Discrete Comput Geom 30:321-336 (2003)

DOI: $10.1007 / \mathrm{s} 00454-003-0013-8$

\title{
A Partitioned Version of the Erdôs-Szekeres Theorem for Quadrilaterals
}

\author{
Attila Pór \\ Rényi Institute of Mathematics, Hungarian Academy of Sciences, \\ PO Box 127, 1364 Budapest, Hungary \\ apor@renyi.hu
}

\begin{abstract}
We prove a partitioned version of the Erdôs-Szekeres theorem for the case $k=4$ : any finite set $X \subset \mathbb{R}^{2}$ of points in general position can be partitioned into sets $X_{0}, X_{i j}$ where $i=1,2,3,4$ and $j=1, \ldots, 26$, so that $\left|X_{1 j}\right|=\left|X_{2 j}\right|=\left|X_{3 j}\right|=\left|X_{4 j}\right|,\left|X_{0}\right| \leq 4$ and for all $j$ every transversal $\left\{x_{1}, x_{2}, x_{3}, x_{4}\right\}, x_{1} \in X_{1 j}, x_{2} \in X_{2 j}, x_{3} \in X_{3 j}, x_{4} \in X_{4 j}$, is in convex position. In order to prove this, we show another theorem, the partitioned version of the "same type lemma", which was proved by Bárány and Valtr in [BV].
\end{abstract}

\section{Introduction}

The Erdôs-Szekeres theorem [ES1], [ES2], [TV] says that among sufficiently many points in general position in the plane one can find $k$ that are in convex position. This classical result in combinatorial geometry has a number of generalizations and extensions (see $[\mathrm{S} 1]$ and $[\mathrm{EP}])$. One of them is a fractional version due to Bárány and Valtr, where they prove:

Theorem [BV]. Given $k \geq 4$, there is a constant $C(k)$ such that for every $X \subset \mathbb{R}^{2}$ of $n$ points in general position the following holds. There are subsets $Y_{1}, \ldots, Y_{k}$ of $X$, each of size at least $C(k) n$ such that for every transversal $y_{1} \in Y_{1}, \ldots, y_{k} \in Y_{k}$, the points $y_{1}, \ldots, y_{k}$ are in convex position.

For $k=4$ this was proved earlier by Nielsen [N]. Solymosi [S2] proved in his master thesis a closely related result, that among $n$ points in the plane there is a sequence of $c_{r} n$ elements such that any $r$ consecutive are in convex position.

The question was raised by Kalai [K1], who asked whether this fractional ErdôsSzekeres theorem admits a partitioned one? We answer this question for $k=4$, which 
is the first interesting case of the problem, with the following result

Theorem 1.1. There exists $N<\infty$, such that if $X \subset \mathbb{R}^{2}$ is a finite set of points in general position, then there is a partition of $X$ into $X_{0}, X_{1,1}, X_{1,2}, X_{1,3}, X_{1,4}, \ldots, X_{N, 1}$, $X_{N, 2}, X_{N, 3}, X_{N, 4}$ such that $\left|X_{0}\right| \leq 4$ and for every fixed $i, 1 \leq i \leq N,\left|X_{i, 1}\right|=\left|X_{i, 2}\right|=$ $\left|X_{i, 3}\right|=\left|X_{i, 4}\right|$ and every transversal $\left\{x_{1}, x_{2}, x_{3}, x_{4}\right\}, x_{j} \in X_{i, j}$, is in convex position, i.e. they form a convex quadrangle.

Remark. We will show that $N=26$ will do at the price of a larger bound on $\left|X_{0}\right|$.

Remark. This result has already been generalized by Valtr in [PV] from quadrilaterals to any $k$-gon in any dimension.

Károlyi investigated a closely related problem: when can a set of $4 n$ points be partitioned into $n$ convex (vertex-disjoint but possibly intersecting) quadrilaterals [K2]?

The proof of Theorem 1.1 (see Section 4) is based on another partitional statement, a generalization of the same type lemma. Given sets $Y_{1}, \ldots, Y_{m}$ a collection $y_{1}, \ldots, y_{m}$, where $y_{i} \in Y_{i}$, is a transversal of the sets $Y_{1}, \ldots, Y_{m}$.

Same Type Lemma [BV]. For any natural numbers $m, d \geq 1$ there exists a constant $c(m, d)$ such that if $X_{1}, \ldots, X_{m} \subset \mathbb{R}^{d}$ are finite sets and $\bigcup_{i} X_{i}$ is in general position, there are subsets $Y_{i} \subset X_{i},\left|Y_{i}\right| \geq c(m, d)\left|X_{i}\right|$ such that every transversal of the sets $Y_{1}, \ldots, Y_{m}$ has the same geometric (order) type.

Pach and Solymosi [PS] generalized the same type lemma for disjoint convex sets.

Each $d+1$ points in general position may have two different orientations, "+" and "-". Here the geometric type means the collection of orientations of the $\left(\begin{array}{c}m \\ d+1\end{array}\right)(d+1)$ tuples, i.e. two point sets of size $m$ have the same geometric type, under a given order of the points, if the $d+1$ points taken from each set from the same $d+1$ positions have the same orientation. We call sets like $Y_{1}, \ldots, Y_{m}$ separated in an "algebraic" sense. We define two other types of separation: "geometric" and "combinatorial".

The sets $X_{1}, \ldots, X_{m}$ are separated in a "geometric" sense, if each hyperplane $H$ intersects at most $d$ sets from conv $X_{1}, \ldots$, conv $X_{m}$.

The number $t_{m, d}$ is the number of bipartitions of $m$ points in $\mathbb{R}^{d}$ in general position by hyperplanes. The sets $X_{1}, \ldots, X_{m}$ are separated in a "combinatorial" sense if the number of bipartitions of the sets by hyperplanes which do not meet any of the sets conv $X_{i}$ is equal to $t_{m, d}$.

We prove in Section 6 that all these definitions are equivalent and therefore call the sets $X_{1}, \ldots, X_{m}$ with either property separated.

To formulate a partitioned version of the same type lemma, we need some further definitions.

We call $M$ a matrix-partition (or $\mu$-partition, for short) with $N$ columns on the sets $X_{1}, \ldots, X_{m} \subset \mathbb{R}^{d}$ if

(i) $M=\left(M_{i j}\right)_{m \times N}, M_{i j} \subset X_{i}$,

(ii) $M_{i j_{1}} \cap M_{i j_{2}}=\emptyset$, for all $i, j_{1}, j_{2}\left(j_{1} \neq j_{2}\right)$,

(iii) $\bigcup_{j} M_{i j}=X_{i}$ for all $i$. 
Combining this with separability, we call the $\mu$-partition $M$ a separated $\mu$-partition if the sets in each column are separated. We call the $\mu$-partition balanced if the sets in the same column have the same size. Clearly, in this case $\left|X_{1}\right|=\cdots=\left|X_{m}\right|$.

Now we can formulate our theorem, generalizing the same type lemma.

Theorem 1.2. For all natural numbers $m, d$ there exists a least integer $n_{m, d}$ such that if finite sets $X_{1}, \ldots, X_{m} \subset \mathbb{R}^{d}$ have the same size and $\bigcup_{i} X_{i}$ is in general position, then there exists a balanced, separated $\mu$-partition with at most $n_{m, d}$ columns on these sets.

We show our theorem by a double induction, based on two lemmas (see Section 2).

The proof of Theorem 1.2 gives an exponential bound in $m$ for $n_{m, 1}$. Better bounds in lower dimensions would yield better bounds in higher dimensions too.

In Section 5 we give asymptotically tight bounds for $n_{m, 1}$, namely

\section{Theorem 1.3.}

$$
\left\lfloor\frac{m-1}{2}\right\rfloor \cdot\left\lfloor\frac{m+1}{2}\right\rfloor+1 \leq n_{m, 1} \leq(m-1)^{2}+1 .
$$

In Section 2 we prove Theorem 1.2 by stating two lemmas. These lemmas are proved in Section 3. The proof of Theorem 1.1 is in Section 4. The proof of Theorem 1.3 is in Section 5 and the equivalence of separability is shown in Section 6.

\section{Proof of the Partitioned "Same Type Lemma"}

Denote by $n_{m, d}$ the smallest number (probably infinite) such that for all finite sets $X_{1}, \ldots, X_{m} \subset \mathbb{R}^{d}$ a balanced separated $\mu$-partition with at most $n_{m, d}$ columns exists. To prove the theorem, we have to show that $n_{m, d}$ is finite, and the following two lemmas will do this.

Lemma 2.1. If $n_{d+1, d}$ is finite for a given dimension $d$, then so are all $n_{m, d}$ numbers, where $m>d+1$ and

$$
n_{m, d} \leq\left(n_{d+1, d}\right)^{\left(\begin{array}{c}
m \\
d+1
\end{array}\right)}
$$

Lemma 2.2. The number $n_{2,1}$ is equal to 2. If $n_{d+1, d-1}$ is finite, then

$$
n_{d+1, d} \leq 2 n_{d+1, d-1} .
$$

Proof of Theorem 1.2. If $n_{d, d-1}$ is finite, then by the first lemma $n_{d+1, d-1}$ is finite too and by the second lemma $n_{d+1, d}$ is also finite. Since $n_{2,1}$ is finite, we get by induction that $n_{d+1, d}$ is finite for all $d$. The first lemma now proves our theorem. 


\section{Proof of the Lemmas}

We will need new notions, the partial $\mu$-partition and the refinement of the $\mu$-partition:

The $\mu$-partition $M$ is partial on the set $I \subset[m]=\{1, \ldots, m\}$ if the matrix $M$ has only entries in the rows $i \in I$ and it satisfies the conditions for $\mu$-partitions in the rows $i \in I$. A partial $\mu$-partition is balanced if the cardinality of the sets in one column is equal, of course only for sets which are in a row with index from $I$. A partial balanced $\mu$-partition can be extended to a balanced $\mu$-partition $M$. For example, partition all other sets $X_{i}, i \notin I$, by taking care only on the cardinality of the entries.

If we have a balanced $\mu$-partition $M$, we can replace some of its columns by a balanced $\mu$-partition of the sets of these columns. Then we get a new balanced $\mu$-partition. We call this the refinement of the $\mu$-partition. Note that the refinement of a refinement of a $\mu$-partition is also a refinement of the original one.

Proof of Lemma 2.1. Let $I_{1}, \ldots, I_{r} \subset[\mathrm{m}]$ be all the subsets of $[\mathrm{m}]$ of $d+1$ elements $\left(r=\left(\begin{array}{c}m \\ d+1\end{array}\right)\right)$. Take the "trivial" $\mu$-partition on the sets with one column, $M_{i 1}=X_{i}, i=$ $1, \ldots, m$, and refine it in several steps, such that after the $l$ th step there will be no hyperplane meeting the convex hulls of the $d+1$ sets $M_{l_{1} j}, \ldots, M_{l_{d+1} j}$ for all $j$, where $I_{l}=\left\{l_{1}, \ldots, l_{d+1}\right\}$.

In the $l$ th step we refine all the columns in the following way: We take the sets in a column and produce a balanced separated $\mu$-partition for the sets in the rows corresponding to $I_{l}$. This is a partial $\mu$-partition for all the sets and has at most $n_{d+1, d}$ new columns which will replace the one column we took. We extend it to a balanced $\mu$-partition and do this refinement for all the columns. At the end we will have a balanced $\mu$-partition, and there will be no hyperplane intersecting the convex hull of any $d+1$ sets in the same column. This means that the balanced $\mu$-partition is separated and the lemma is proved since in each of the $r$ steps the size of the matrix was multiplied by at most $n_{d+1, d}$.

In Lemma 2.2 first we prove the starting step $(d=1)$ and then the induction.

Proof of Lemma 2.2. Let $X_{1}, X_{2} \subset \mathbb{R},\left|X_{1}\right|=\left|X_{2}\right|=l$, then there is a point $a \in \mathbb{R}$ such that

$$
\left|\left\{x \mid x \in X_{1} \cup X_{2} \quad x \leq a\right\}\right|=l
$$

and define

$$
\begin{aligned}
& M_{11}=\left\{x \in X_{1} \mid x \leq a\right\}, \\
& M_{12}=\left\{x \in X_{1} \mid x>a\right\}, \\
& M_{21}=\left\{x \in X_{2} \mid x>a\right\}, \\
& M_{22}=\left\{x \in X_{2} \mid x \leq a\right\} .
\end{aligned}
$$

Now $M$ is a $\mu$-partition with two columns on the sets $X_{1}$ and $X_{2}$ and

$$
\left|M_{11}\right|+\left|M_{12}\right|=\left|M_{11}\right|+\left|M_{22}\right|=\left|M_{21}\right|+\left|M_{22}\right|=l,
$$

which shows that $M$ is balanced and separated, too. 
In the second part of the lemma we have to show that $n_{d+1, d} \leq 2 n_{d+1, d-1}$ assuming $n_{d+1, d-1}$ is finite. Let $X_{1}, \ldots, X_{d+1} \subset \mathbb{R}^{d}$ be finite sets of size $l$ and suppose that the set $\bigcup_{i} X_{i}$ is in general position. It is well known that there is a hyperplane $S$ such that the perpendicular projection of $\bigcup_{i} X_{i}$ to $S$ is still in general position (in fact, almost all hyperplanes satisfy this condition). Let $X_{i}^{\prime}$ be the image of $X_{i}$ under this projection, and take a balanced separated $\mu$-partition $M^{\prime}$ on these sets, with at most $n_{d+1, d-1}$ columns. Let $M$ be the balanced $\mu$-partition on the sets $X_{1}, \ldots, X_{d+1}$ induced by $M^{\prime}$ and from now on we examine only one of its columns, and for simplicity of notation we now call these sets $X_{1}, \ldots, X_{d+1}$. To prove our lemma, we have to divide all the sets $X_{1}, \ldots, X_{d+1}$ into two parts in a balanced way such that both parts are separated. The projection of these sets are separated in the hyperplane $S$. By Radon's theorem there is only one partition of $d+1$ points in $\mathbb{R}^{d-1}$, among all the $2^{d}$, which cannot be separated by a hyperplane, which means, in combinatorial sense, that there are $2^{d}-1$ hyperplanes in $S$ which miss $\bigcup_{i} \operatorname{conv} X_{i}^{\prime}$ and divide them in different ways. Take for each hyperplane in $S$ the hyperplane in $\mathbb{R}^{d}$ as its inverse image under the perpendicular projection to $S$. These hyperplanes avoid $\bigcup_{i} X_{i}$ and divide them in $2^{d}-1$ different ways. Since there are $2^{d}$ different bipartitions of the set $[d+1]$, there is one remaining bipartition $\left(I \subset[d+1], I^{c}=[d+1] \backslash I\right)$ for which we need separability.

For technical reasons it will be useful to change the point-set to a measurable set which is close to the point-set in the following way: Replace all points by a ball centered at the point and of radius $\varepsilon$, and denote the new sets by $K_{1}, \ldots, K_{d+1}$. Since $\bigcup_{i} X_{i}$ is in general position it is possible to choose $\varepsilon$ so small that no hyperplane can meet $d+1$ of the balls.

Now we assume that the measure is distributed uniformly on these balls, such that each of them has measure one (i.e. we divide the Lebesgue measure by the volume of the ball of radius $\varepsilon$ and denote it by $v$ ). We define a function $f: S^{d} \rightarrow \mathbb{R}^{d}$ which is odd. Take $H=(t, \mathbf{h}) \in S^{d}$, where $t$ is a real number and $\mathbf{h}$ is a $d$-dimensional vector not equal to zero. Let

$$
H_{i}^{+}=\{x \mid\langle x, \mathbf{h}\rangle \geq t\} \cap K_{i}
$$

and

$$
H_{i}^{-}=\{x \mid\langle x, \mathbf{h}\rangle<t\} \cap K_{i}=K_{i} \backslash H_{i}^{+} .
$$

Further let

$$
H_{i}^{*}= \begin{cases}H_{i}^{+} & \text {if } \quad i \in I, \\ H_{i}^{-} & \text {if } \quad i \notin I .\end{cases}
$$

Now we can define $f$ at $(t, \mathbf{h})$ as follows:

$$
f((t, \mathbf{h}))=\left(v\left(H_{1}^{*}\right)-v\left(H_{d+1}^{*}\right), \ldots, v\left(H_{d}^{*}\right)-v\left(H_{d+1}^{*}\right)\right) \in \mathbb{R}^{d} .
$$

It is easy to check that $(-H)_{i}^{+}=H_{i}^{-}$, and we can extend the definition to $H=(+1, \mathbf{0})$ by choosing

$$
(+1, \mathbf{0})_{i}^{*}=H_{i}^{*}= \begin{cases}\emptyset & \text { if } \quad i \in I, \\ K_{i} & \text { if } \quad i \notin I,\end{cases}
$$


and for $H=(-1, \mathbf{0})$,

$$
(-1, \mathbf{0})_{i}^{*}=H_{i}^{*}= \begin{cases}K_{i} & \text { if } \quad i \in I, \\ \varnothing & \text { if } i \notin I .\end{cases}
$$

The function $f$ maps $S^{d}$ into $\mathbb{R}^{d}$ and it is continuous and odd so Borsuk's theorem applies: there exists an $H \in S^{d}$ (of course not one of the poles) such that $f(H)=\mathbf{0}$. The hyperplane $S_{H}=\{x \mid\langle x, \mathbf{h}\rangle=t\}$ meets at most $d$ balls of radius $\varepsilon$ with a center in $\bigcup_{i} X_{i}$, therefore one of the sets $K_{j}$ has been avoided by $S_{H}$ and so $v\left(H_{j}^{*}\right)=r$ is an integer number and a hyperplane $T$ (a slight perturbation of the one corresponding to $H$ ) can be chosen such that it does not meet $\bigcup_{i} X_{i}$ and on a given side it contains $r$ points from $X_{i}$ if $i \in I$, otherwise it contains $l-r$ points. If $T^{+}$denotes one half-space generated by $T$, then the $\mu$-partition of two columns are now the subsets of $X_{i} \cap T^{+}$if $i \in I$ and $X_{j} \backslash T^{+}$if $j \notin I$. The properties of $H$ show that this partition is balanced and separated.

Remark. If $d=2$ the above proof gives the following statement that will be needed in the next section: If three equal-sized sets $X_{1}, X_{2}$ and $X_{3}$ are separated by two parallel lines in the plane, then there exists a line $f$ which divides the sets into $X_{i 1}$ and $X_{i 2}$ for $i=1,2,3$, such that $X_{11}, X_{22}$ and $X_{31}$ are on the same side of $f$ and

$$
\frac{\left|X_{11}\right|}{\left|X_{12}\right|}=\frac{\left|X_{21}\right|}{\left|X_{22}\right|}=\frac{\left|X_{31}\right|}{\left|X_{32}\right|}
$$

are equal (probably infinite).

\section{Proof of Theorem 1.1}

Observe that among any five points in the plane there are four in convex position, and therefore it would be enough to bound $X_{0}$ by a finite number, since then we can remove four points in convex position, and increase $N$ by one, adding a column with four sets of one element to our partition.

Proof of Theorem 1.1. Let us divide $X$ into four equal sets $X_{1}, X_{2}, X_{3}$ and $X_{4}$ by dropping at most three points. By Theorem 1.2 there exists a balanced, separated $\mu$ partition $M$ with at most $n_{4,2}$ columns. Some columns of this matrix are already in convex position, and we can use them as they are in our decomposition. So we can reduce further investigations to a given column, where the sets are not in convex position. From now on we suppose that $X=\bigcup_{i}^{4} X_{i}$, where $X_{1}, X_{2}, X_{3}, X_{4}$ are separated and of equal size, say $2 l$, and for any transversal $\left\{x_{1}, x_{2}, x_{3}, x_{4}\right\}, x_{i} \in X_{i}$, the point $x_{4}$ is in the convex hull of the other three, $x_{4} \in \operatorname{conv}\left\{x_{1}, x_{2}, x_{3}\right\}$. The assumption of even size enlarges $X_{0}$. Because the sets are separated, there are lines $a_{1}, a_{2}$ and $a_{3}$, such that on one side of $a_{i}$ is $X_{i}, X_{4}$ and all other sets $X_{j}, j \neq i, 4$, are on the opposite side. Now choose the lines $e_{1}, e_{2}$ and $e_{3}$, each parallel to the corresponding line $a_{i}$, such that $e_{i}$ divides the set $X_{i}$ into two sets of equal size, $X_{i 1}$ and $X_{i 2}$, with $X_{i 2}$ on the same side of $e_{i}$ as $X_{4}$. Clearly, there exists a line $l_{i}$ parallel to $a_{i}$ and $e_{i}$ separating $X_{i}$ from $X_{4}$. Now the two parallel lines $e_{i}$ and $l_{i}$ 
separate $X_{i 1}, X_{i 2}$ and $X_{4}$. We denote by $2 S$ the set derived from $S$ by doubling and slightly perturbing its points. Now choose the line $f_{i}$ to divide the equal-sized sets $2 X_{i 1}, 2 X_{i 2}$ and $X_{4}$ as in the Remark of the previous section. Since we doubled some of the sets, we will need some correction at the end, but this means only a constant in the size of $X_{0}$.

Let $X_{4 i}^{\prime}, X_{4 i}^{\prime \prime}$ be the two parts of $X_{4}$ divided by $f_{i}$. Corresponding to the previous notation, let $X_{i 1}^{\prime}, X_{i 1}^{\prime \prime}, X_{i 2}^{\prime}, X_{i 2}^{\prime \prime}$ be the sets derived from the sets $X_{i 1}, X_{i 2}$ after dividing them by $f_{i}$, i.e.

$$
\begin{aligned}
& \alpha=\frac{\left|X_{11}^{\prime}\right|}{\left|X_{11}\right|}=\frac{\left|X_{12}^{\prime}\right|}{\left|X_{12}\right|}=\frac{\left|X_{41}^{\prime}\right|}{\left|X_{4}\right|}, \\
& \beta=\frac{\left|X_{21}^{\prime}\right|}{\left|X_{21}\right|}=\frac{\left|X_{22}^{\prime}\right|}{\left|X_{22}\right|}=\frac{\left|X_{42}^{\prime}\right|}{\left|X_{4}\right|}, \\
& \gamma=\frac{\left|X_{31}^{\prime}\right|}{\left|X_{31}\right|}=\frac{\left|X_{32}^{\prime}\right|}{\left|X_{32}\right|}=\frac{\left|X_{43}^{\prime}\right|}{\left|X_{4}\right|} .
\end{aligned}
$$

It is easy to see that the following 4-tuples of sets are separated and in convex position (Fig. 1.):

$$
\begin{array}{lllll}
X_{11}^{\prime}, & X_{12}^{\prime}, & X_{41}^{\prime}, & X_{2} & \left(\lambda_{1}\right) \\
X_{11}^{\prime \prime}, & X_{12}^{\prime \prime}, & X_{41}^{\prime \prime}, & X_{3} & \left(\lambda_{1}\right) \\
X_{21}^{\prime}, & X_{22}^{\prime}, & X_{42}^{\prime}, & X_{3} & \left(\lambda_{2}\right) \\
X_{22}^{\prime \prime}, & X_{22}^{\prime \prime}, & X_{42}^{\prime \prime}, & X_{1} & \left(\lambda_{2}\right) \\
X_{313}^{\prime}, & X_{32}^{\prime}, & X_{43}^{\prime}, & X_{1} & \left(\lambda_{3}\right) \\
X_{31}^{\prime \prime}, & X_{32}^{\prime \prime}, & X_{43}^{\prime \prime}, & X_{2} & \left(\lambda_{3}\right)
\end{array}
$$

For example choose the first 4-tuple, $X_{11}^{\prime}, X_{12}^{\prime}, X_{41}^{\prime}, X_{2}$. One can see on Fig. 1 that the line $e_{1}$ separates $X_{11}^{\prime}$ from the other three sets. Further, the lines $f_{1}, a_{3}, a_{1}$ in order separate $X_{12}^{\prime}, X_{41}^{\prime}$ and $X_{2}$ from the remaining three sets.

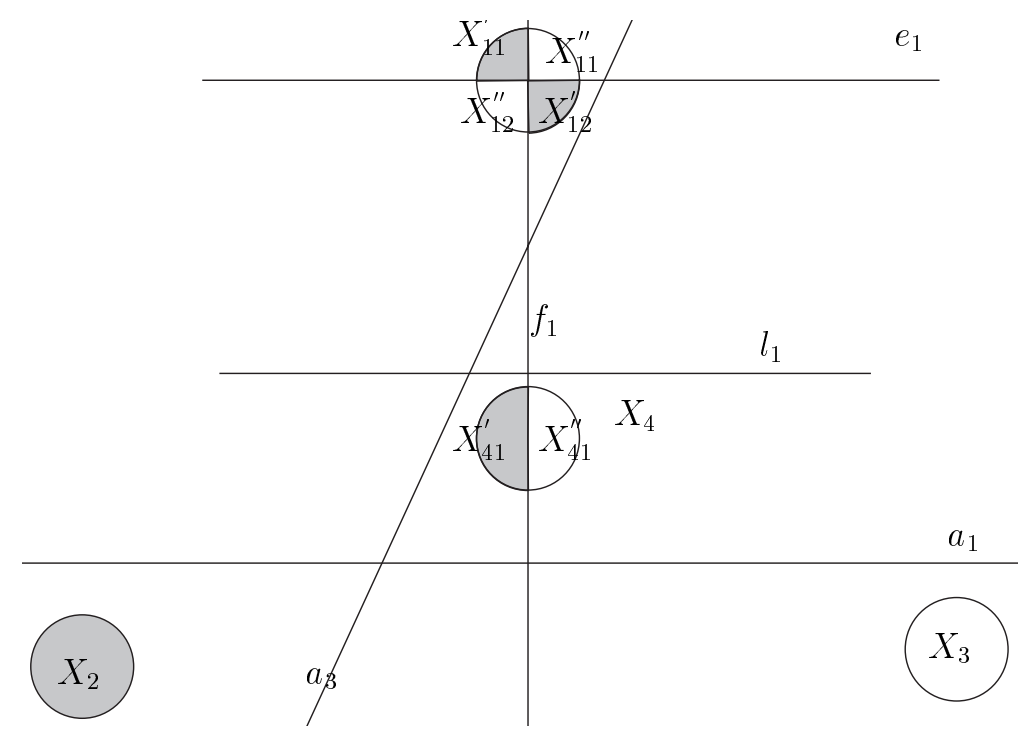

Fig. 1. Nonconvex 4-tuple. 
These six 4-tuples cover the set $X$ several times, so we need to reduce them so that they give a partition of $X$. The four sets of one 4-tuple are not equal sized, therefore we prescribe the size of the sets in order by $\lambda_{1}\left|X_{11}^{\prime}\right|, \lambda_{1}\left|X_{11}^{\prime \prime}\right|, \lambda_{2}\left|X_{21}^{\prime}\right|, \lambda_{2}\left|X_{21}^{\prime \prime}\right|, \lambda_{3}\left|X_{31}^{\prime}\right|, \lambda_{3}\left|X_{31}^{\prime \prime}\right|$. To construct the sets themselves, we have to check first that at least the size of the sets $X_{i}$ are as big as the sum of its parts. Therefore we will get four equalities for the ratios $\lambda_{1}, \lambda_{2}, \lambda_{3}$ which have to be fulfilled in order to continue the construction. We denote the subsets by $Y_{* *}$ with subscripts like the corresponding $X_{* *}$ sets, except $Y_{i}^{\prime}$ and $Y_{i}^{\prime \prime}$ for $i=1,2,3$ which are the fourth sets of the 4-tuples listed above. First we calculate their sizes, in order $\lambda_{1} \alpha l, \lambda_{1}(1-\alpha) l, \lambda_{2} \beta l, \lambda_{2}(1-\beta) l, \lambda_{3} \gamma l, \lambda_{3}(1-\gamma) l$, where $0 \leq \lambda_{i} \leq 1$ for all $i=1,2,3$ :

$$
\begin{aligned}
\lambda_{1} \alpha l & =\left|Y_{11}^{\prime}\right|=\left|Y_{12}^{\prime}\right|=\left|Y_{41}^{\prime}\right|=\left|Y_{2}^{\prime}\right|, \\
\lambda_{1}(1-\alpha) l & =\left|Y_{11}^{\prime \prime}\right|=\left|Y_{12}^{\prime \prime}\right|=\left|Y_{41}^{\prime \prime}\right|=\left|Y_{3}^{\prime \prime}\right|, \\
\lambda_{2} \beta l & =\left|Y_{21}^{\prime}\right|=\left|Y_{22}^{\prime}\right|=\left|Y_{42}^{\prime}\right|=\left|Y_{3}^{\prime}\right|, \\
\lambda_{2}(1-\beta) l & =\left|Y_{21}^{\prime \prime}\right|=\left|Y_{22}^{\prime \prime}\right|=\left|Y_{42}^{\prime \prime}\right|=\left|Y_{1}^{\prime \prime}\right|, \\
\lambda_{3} \gamma l & =\left|Y_{31}^{\prime}\right|=\left|Y_{32}^{\prime}\right|=\left|Y_{43}^{\prime}\right|=\left|Y_{1}^{\prime}\right|, \\
\lambda_{3}(1-\gamma) l & =\left|Y_{31}^{\prime \prime}\right|=\left|Y_{32}^{\prime \prime}\right|=\left|Y_{43}^{\prime \prime}\right|=\left|Y_{2}^{\prime \prime}\right| .
\end{aligned}
$$

Since

$$
\begin{aligned}
& X_{1}=Y_{11}^{\prime} \cup Y_{11}^{\prime \prime} \cup Y_{12}^{\prime} \cup Y_{12}^{\prime \prime} \cup Y_{1}^{\prime} \cup Y_{1}^{\prime \prime}, \\
& X_{2}=Y_{21}^{\prime} \cup Y_{21}^{\prime \prime} \cup Y_{22}^{\prime} \cup Y_{22}^{\prime \prime} \cup Y_{2}^{\prime} \cup Y_{2}^{\prime \prime}, \\
& X_{3}=Y_{31}^{\prime} \cup Y_{31}^{\prime \prime} \cup Y_{32}^{\prime} \cup Y_{32}^{\prime \prime} \cup Y_{3}^{\prime} \cup Y_{3}^{\prime \prime}, \\
& X_{4}=Y_{41}^{\prime} \cup Y_{41}^{\prime \prime} \cup Y_{42}^{\prime} \cup Y_{42}^{\prime \prime} \cup Y_{43}^{\prime} \cup Y_{43}^{\prime \prime},
\end{aligned}
$$

where each line is a partition of the corresponding $X_{i}$, we get four equalities for the sizes (each equation divided by $l$ )

$$
\begin{aligned}
& 2=2 \lambda_{1}+\lambda_{2}(1-\beta)+\lambda_{3} \gamma, \\
& 2=2 \lambda_{2}+\lambda_{3}(1-\gamma)+\lambda_{1} \alpha, \\
& 2=2 \lambda_{3}+\lambda_{1}(1-\alpha)+\lambda_{2} \beta=4-\lambda_{1}(1+\alpha)-\lambda_{2}(2-\beta), \\
& 2=\lambda_{1}+\lambda_{2}+\lambda_{3} .
\end{aligned}
$$

The solution is

$$
\begin{aligned}
& \lambda_{1}=\frac{2+2 \beta \gamma-2 \gamma}{3+\alpha \beta+\beta \gamma+\gamma \alpha-\alpha-\beta-\gamma}, \\
& \lambda_{2}=\frac{2+2 \gamma \alpha-2 \alpha}{3+\alpha \beta+\beta \gamma+\gamma \alpha-\alpha-\beta-\gamma}, \\
& \lambda_{3}=\frac{2+2 \alpha \beta-2 \beta}{3+\alpha \beta+\beta \gamma+\gamma \alpha-\alpha-\beta-\gamma},
\end{aligned}
$$

where we can check that $0 \leq \lambda_{1}, \lambda_{2}, \lambda_{3} \leq 1$. 
Now the sizes of our sets $Y_{11}^{\prime}, \ldots, Y_{43}^{\prime \prime}$ are known, but how to choose them? Take the set $X_{1}$ and choose first the sets $Y_{11}^{\prime}, Y_{11}^{\prime \prime}, Y_{12}^{\prime}, Y_{12}^{\prime \prime}$ and the remaining points from $X_{1}$ can be distributed between $Y_{1}^{\prime}$ and $Y_{1}^{\prime \prime}$ arbitrarily. The distribution of $X_{2}$ and $X_{3}$ are similar. To distribute the points of $X_{4}$ we take the arrangement of the lines $f_{1}, f_{2}$ and $f_{3}$ which partitions the set $X_{4}$ into at most seven parts. In each part we distribute the points into three parts of size $\lambda_{1}: \lambda_{2}: \lambda_{3}$. It is clear that we can put together the sets $Y_{41}^{\prime}, \ldots, Y_{43}^{\prime \prime}$ from these parts.

During these steps we do not care about some (finitely many) points which do not satisfy our partition requirements. As we doubled some points for example, at the end we probably have to remove one point from a set. Since these are just finitely many points they may be appended to $X_{0}$.

Remark. This immediately gives a bound of $6 n_{4,2}$ but we can obtain 26 .

Lemma 4.1. Theorem 1.1 is true with $N=26$.

Proof. We change each point of $X$ to a nowhere zero measure distributed on the plane, concentrated on a disc centered at the point (similarly to the proof of Lemma 2.2). One can achieve this by distributing $\varepsilon$ measure outside a disc of radius $\varepsilon$.

Choose an arbitrary directed line $l$ and divide $X$ into four equal parts $X_{1}, X_{2}, X_{3}$ and $X_{4}$ by three lines $l_{1}, l_{2}, l_{3}$ all parallel to $l$ (Fig. 2).

Take the vertical line as $l$, such that the direction points upwards. For each $0 \leq \varepsilon \leq 1$ there is a well defined half-space $H_{\varepsilon}$ with boundary line $H_{\varepsilon}^{0}$ for which

$$
\frac{\left|H_{\varepsilon} \cap X_{2}\right|}{\left|X_{2}\right|}=1-\varepsilon \quad \text { and } \quad \frac{\left|H_{\varepsilon} \cap X_{3}\right|}{\left|X_{3}\right|}=\varepsilon
$$

and $H_{\varepsilon}$ is below $H_{\varepsilon}^{0}$. Let

$$
\begin{aligned}
& \beta(\varepsilon)=\frac{\left|H_{\varepsilon} \cap X_{1}\right|}{\left|X_{1}\right|}, \\
& \gamma(\varepsilon)=1-\frac{\left|H_{\varepsilon} \cap X_{4}\right|}{\left|X_{4}\right|} .
\end{aligned}
$$

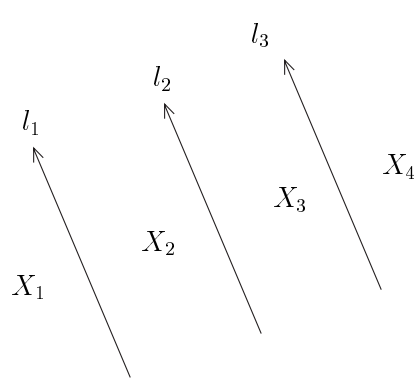

Fig. 2. Parallel 4-partitioned set. 


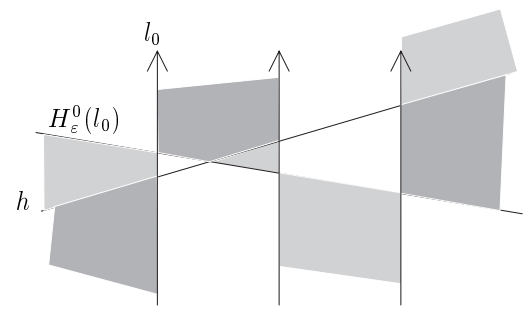

Fig. 3. The right line.

Clearly, $\beta(0)=\gamma(0)=1, \beta(1)=\gamma(1)=0$ and $\beta, \gamma$ are continuous functions in $\varepsilon$. Let

$$
\varepsilon(l)=\min _{\varepsilon}\{\min \{\beta(\varepsilon), \gamma(\varepsilon)\} \leq \varepsilon\} .
$$

We are looking for a line $l$ with

$$
\begin{aligned}
& \beta(\varepsilon(l))=\varepsilon(l), \\
& \gamma(\varepsilon(l))=\varepsilon(l) .
\end{aligned}
$$

At least one of the two equalities holds for every line, so turning $l$ around (by $180^{\circ}$, at the end the direction of the starting line has been changed) we get that the roles of $\beta$ and $\gamma$ change. Since $\varepsilon(l)$ was a continuous function therefore we had to pass a line $l_{0}$ for which

$$
\beta\left(\varepsilon\left(l_{0}\right)\right)=\varepsilon\left(l_{0}\right) \quad \text { and } \quad \gamma\left(\varepsilon\left(l_{0}\right)\right)=\varepsilon\left(l_{0}\right) .
$$

Choosing this line and the corresponding lines $l_{1}, l_{2}, l_{3}$ and $H_{\varepsilon}^{0}\left(l_{0}\right)$ (Fig. 3) we get a partition of $X$ into the sets $Z_{1}, \ldots, Z_{4}, Y_{1}, \ldots, Y_{4}$, where $X_{1}=Z_{1} \cup Y_{1}, \ldots, X_{4}=$ $Z_{4} \cup Y_{4}$. The shadowed parts are the $Z_{i}$ and the white parts the $Y_{i}$ sets by indexing them from left to right.

Now separating $Z_{1}, Z_{2}, Z_{4}$ by $h$ as in the Remark at the end of Section 3 we get two parts. The light shadow part by adding a part of $Z_{3}$ to it, is already a separated 4-tuple (nonconvex). Doing the same for $Z_{1}, Z_{3}, Z_{4}$ we get again a nonconvex separated 4-tuple and the rest is a convex separated 4-tuple. The previous theorem replaces the nonconvex ones by $2 \cdot 6=12$ convex parts. The same is true for the sets $Y_{1}, \ldots, Y_{4}$ and we get at most 26 convex 4-tuples.

\section{Asymptotically Tight Bounds for $n_{m, 1}$}

From our previous proofs we know that $n_{2,1}=2$, and that

$$
n_{m, 1} \leq\left(n_{2,1}\right)^{\left(\begin{array}{c}
m \\
2
\end{array}\right)}=2^{m(m-1) / 2}
$$

which is super-exponential in $m$, and even for $m=3$ it gives an upper bound of $n_{3,1} \leq 8$ while the truth should be $n_{3,1}=5$ (easy as it seems, I could not prove it). We will prove a qudratic upper bound on $n_{m, 1}$ and show that it is the truth up to a constant factor. 
We prove Theorem 1.3 in two steps.

Let us recall the upper bound of Theorem 1.3:

If $X_{1}, \ldots, X_{m}$ are finite sets of equal size on the real line and the set $\bigcup_{i}^{m} X_{i}$ is in general position, then there is a balanced, separated $\mu$-partition with at most $(m-1)^{2}+1$ columns on these sets, i.e.

$$
n_{m, 1} \leq(m-1)^{2}+1 .
$$

Proof of the upper bound. Let $X=\bigcup_{i}^{m} X_{i}$, and $l=\left|X_{i}\right|$ be the size of the sets. Take $m-1$ points, $t_{1}, \ldots, t_{m-1}$, on the real line which partitions the set $X$ into $m$ equal sets $T_{1}, \ldots, T_{m}$ :

$$
-\infty=t_{0}<t_{1}<\cdots<t_{m-1}<t_{m}=\infty
$$

and for all $1 \leq i, j \leq m$,

$$
T_{j}=\left\{x \in X \mid t_{j-1} \leq x<t_{j}\right\}
$$

Take the intersection of these sets with the sets $X_{i}$ and denote them for all $1 \leq i, j \leq m$ by

$$
A_{i, j}=X_{i} \cap T_{j} .
$$

Define the matrix $S$ of size $m \times m$ with integer entries:

$$
S_{i, j}=\left|A_{i, j}\right| .
$$

The matrix $S$ has the property that the sum of its entries in a row or a column are equal (constant times a doubly stochastic matrix), and all of its entries are nonnegative. We call such a nonnegative matrix a "magic matrix". We know (see the Remark below or [MM]) that $S$ is a positive integer combination of permutation matrices $P_{1}, \ldots, P_{k}$, where $k \leq(m-1)^{2}+1$,

$$
S=\sum_{i=1}^{k} \lambda_{i} P_{i},
$$

where $\lambda_{i}$ is a positive integer. Let $\sigma_{i}$ be the permutation of $P_{i}$. Now we can construct our $\mu$-partition $M$ of $k$ elements:

$$
M_{i, j} \subset A_{i, \sigma_{i}(j)} \quad \text { and } \quad\left|M_{i, j}\right|=\lambda_{i}
$$

and such $M$ exists. (We do not care about how these subsets are chosen, just that they have to be disjoint.)

It is clear that $M$ is balanced and separated. The points $t_{1}, \ldots, t_{m-1}$ work as separators for each column.

Remark. We show that an $m \times m$ magic matrix can be written as a positive linear integer combination of at most $m^{2}-2 m+2$ permutation matrices.

All matrices here will have positive integer entries. 
First we show that for each magic matrix $D$, there exists a permutation matrix $P$, such that $P \leq D$.

Suppose that such a $P$ permutation matrix does not exist. We know (see [MM]) that $D$ has an $m_{1} \times m_{2}$ submatrix, whose entries are all zero and $m_{1}+m_{2}>m$. We may suppose that $D_{i, j}=0$ whenever $i>m-m_{1}$ and $j>m-m_{2}$. If $T$ denotes the sum of entries in one row of $D$, then

$$
T \cdot m_{1}=\sum_{i=m-m_{1}+1}^{m} \sum_{j=1}^{k} D_{i, j}=\sum_{i=m-m_{1}+1}^{m} \sum_{j=1}^{m-m_{2}} D_{i, j} \leq \sum_{i=1}^{m} \sum_{j=1}^{m-m_{2}} D_{i, j}=T \cdot\left(m-m_{2}\right),
$$

which yields

$$
m_{1}+m_{2} \leq m
$$

This is a contradiction to $m_{1}+m_{2}>m$.

Now choose one of the permutation matrices $P_{1} \leq D$ and choose $a_{1}$ such that

$$
a_{1} P_{1} \leq D
$$

but

$$
\left(a_{1}+1\right) P_{1} \not \leq D .
$$

Let $D_{2}=D-a_{1} P_{1}$, and observe that $D_{2}$ is a magic matrix again, which has a zero entry, where $D$ has a nonzero $\left(a_{1}\right)$ entry. Do this as long $D_{i}$ is not the 0 -matrix and we get

$$
D=\sum_{i=0}^{k} a_{i} P_{i}
$$

a positive linear combination of $k$ permutation matrices. Claim: $P_{1}, \ldots, P_{k}$ are linearly independent. Indeed, if they were not, then some $P_{j}$ can be written as a linear combination of $P_{i}$ 's, where $i>j$. When we have choosen $P_{j}$ we nullified at least one entry of the matrix $D_{j}$ and therefore all $P_{i}, i>j$, have a zero value at that entry. This contradiction shows that $P_{1}, \ldots, P_{k}$ are linear independent permutation matrices, and therefore $k$ is at most the dimension of the space generated by permutation matrices, which is $m^{2}-2 m+2$.

I conjecture that this is the truth in one-dimension, but could only prove a lower bound which is four times smaller. It is enough to work for $m=2 r+1$.

Recall the lower bound of Theorem 1.3:

For each odd $m=2 r+1$ there are finite sets $X_{1}, \ldots, X_{m}$ of equal size on the real line, such that their union is in general position and for all balanced separated $\mu$-partitions, the number of columns is at least $r^{2}+r+1$.

In the previous proof we used the term "magic matrix". The role of the magic matrix will be taken by a "nice matrix". A $d$ matrix is nice by definition, if the sum of each row is equal.

The role of permutation matrices will be taken by "special matrices" which are $0-1$ matrices with exactly one nonzero entry in each row. 
Proof of the lower bound. The general idea of the proof is to fix a structure of the sets, and derive a nonnegative $(r+1) \times(r+1)$ nice matrix for them, i.e. the sum of the entries is the same in each row. In this fixed structure of the sets the columns of the $\mu$-partitions will correspond to an integer multiple of an $(r+1) \times(r+1)$ special matrix, and from the definition we will get that the nice matrix can be written as an integer linear combination of $k$ special matrices, where $k=n_{m, 1}$. The next step will be to show that if every nice integer $(r+1) \times(r+1)$ matrix can be written as positive integer linear combination of at most $k$ special matrices, then all nice real $(r+1) \times(r+1)$ matrices can be written as a positive linear combination of at most $k$ special matrices. In the last step we only have to show that if each nice $(r+1) \times(r+1)$ matrix can be written as a positive linear combination of at most $k$ special matrices, than $k$ cannot be less than $r^{2}+r+1$, the dimension of the space of nice $(r+1) \times(r+1)$ matrices.

In general for $1 \leq t \leq r$ we choose the sets $X_{r+1+t}$ so that all of its points are in a small $\varepsilon$ neighborhood of $t([t-\varepsilon, t+\varepsilon])$. The points of the other sets, $X_{j}$, where $1 \leq j \leq r+1$, will be at most $\varepsilon$ distance from a point $z+\frac{1}{2}$ where $z$ is a nonnegative integer number at most $r\left(z=0, \ldots, r\right.$, and $\varepsilon$ can be chosen as $\left.\frac{1}{6}\right)$, i.e. $X_{j} \subset \bigcup_{z=0}^{r}\left[z+\frac{1}{2}-\varepsilon, z+\frac{1}{2}+\varepsilon\right]$. For all $1 \leq i, j \leq r+1$, let

$$
A_{i, j}=X_{i} \cap[j-1, j]
$$

and

$$
d_{i, j}=\left|A_{i, j}\right| .
$$

The matrix $d=\left(d_{i j}\right)$ is the nice integer matrix corresponding to the sets.

Suppose now that $M$ is a $\mu$-partition with $k$ columns of the sets $X_{1}, \ldots, X_{m}$, and take one of its columns, for example, $M_{1,1}, \ldots, M_{1, m}$. The sets $M_{1, r+1+t}$, where $1 \leq t \leq r$, are in an $\varepsilon$ neighborhood of $t, M_{1, r+1+t} \subset X_{r+1+t} \subset[t-\varepsilon, t+\varepsilon]$, and the sets $M_{1, j} \subset X_{j} \subset \bigcup_{z=0}^{r}\left[z+\frac{1}{2}-\varepsilon, z+\frac{1}{2}+\varepsilon\right]$ cannot have elements in the neighborhood of two different points of the form $z+\frac{1}{2}, z \in \mathbb{N}$. This is clear, since otherwise the convex hull of $M_{1, j}$ would contain an integer $t^{\prime}$ between 1 and $r, t^{\prime} \in \operatorname{conv} M_{1, j}$, and therefore it also contains its neighborhood $\left[t^{\prime}-\frac{1}{2}+\varepsilon, t^{\prime}+\frac{1}{2}-\varepsilon\right] \supset\left[t^{\prime}-\varepsilon, t^{\prime}+\varepsilon\right]$, which contains $M_{1, r+1+t^{\prime}}$. This contradicts that the sets $M_{1,1}, \ldots, M_{1, m}$ are separated.

Denote by $i_{j}$ the integer for which $M_{1, j} \subset\left[i_{j}+\frac{1}{2}-\varepsilon, i_{j}+\frac{1}{2}+\varepsilon\right]$, and $1 \leq i_{j} \leq r+1$.

Now we know that $M_{1, j} \subset A_{j, i_{j}}$ for $1 \leq j \leq r+1$. Let $S_{1}$ be the matrix where the $(j, i)$ th entry is the cardinality of the set $M_{1, j} \cap[i-1, i]$. As shown above, the matrix $S_{1}$ is an integer multiple of a special matrix, the one corresponding to the first column. However, so are all matrices $S_{l}$ corresponding to the $l$ th column, where $1 \leq l \leq k$, and therefore the matrix $d=\left(d_{i, j}\right)_{r+1 \times r+1}$ is a positive linear combination of at most $k$ special matrices.

Given an $(r+1) \times(r+1)$ nice integer matrix $d$, we can easily choose $m$ sets so that the nice matrix derived from them is $d$, and therefore all nice integer matrices can be written as a positive integer combination of at most $k$ special matrices.

Let $D=(D(i, j))$ be a nice positive real matrix and define

$$
n_{0}=\frac{2 r}{\min _{2 \leq i \leq r+1} D(i, r+1)} .
$$


We define a sequence of nice integer matrices as follows:

$$
D_{N}=\lfloor N \cdot D\rfloor,
$$

where $N \in \mathbb{N}$ and $N \geq n_{0}$, such that for all $1 \leq j \leq r$ and $i=1, j=r+1$,

$$
D_{N}(i, j)=\lfloor N D(i, j)\rfloor .
$$

All other entries $(j=r+1,2 \leq i \leq r+1)$ of the matrix $D_{N}$ are determined since $D_{N}$ should be a nice matrix. According to rounding

$$
\left|D_{N}(i, r+1)-\lfloor N D(i, r+1)\rfloor\right| \leq r+1
$$

and so the matrix $D_{N}$ will be a nice positive integer matrix for every $N \geq n_{0}$. Further, as $N \rightarrow \infty$,

$$
\frac{D_{N}}{N} \rightarrow D
$$

and all matrices $D_{N}\left(N \geq n_{0}\right)$ can be written as a positive linear combination of at most $k$ special matrices:

$$
D_{N}=\sum_{i}^{k} a_{i}^{N} S_{i}^{N}, \quad \text { where } \quad a_{i}^{N} \in N .
$$

Since there are only finitely many special matrices (exactly $(r+1)^{r+1}$ ) we can choose a subsequence of $D_{N}$, namely $D_{N_{j}}$, where $S_{i}^{N_{j}}=S_{i}^{N_{j^{\prime}}}$ for all $i, j, j^{\prime} \in N$. Denote the common special matrix by $S_{i}=S_{i}^{N_{1}}$. Further, the sequence

$$
\frac{a_{i}^{N_{1}}}{N_{1}}, \frac{a_{i}^{N_{2}}}{N_{2}}, \ldots
$$

is bounded, and therefore we can even choose a subsequence that is convergent for all $1 \leq i \leq k$, and has as limit $\beta_{i}$ :

$$
\lim _{j} \frac{a_{i}^{N_{j}}}{N_{j}}=\beta_{i}
$$

where $\beta_{i}$ is of course nonnegative.

A simple limit argument shows that

$$
D=\sum_{i}^{k} \beta_{i} S_{i},
$$

so all positive real nice matrices $D$ can be written as a nonnegative linear combination of at most $k$ special matrices. As there are finitely many $k$-tuples of special matrices, one of them will cover an open set of the space of positive real nice matrices, and therefore the linear combinations over these $k$ matrices generate the space of the nice matrices, which has dimension $r^{2}+r+1$. This proves our lemma that

$$
n_{m, 1} \geq k \geq r^{2}+r+1 \text {. }
$$




\section{Equivalence of Three Definitions about Separated Sets}

Lemma 6.1 [GP1], [GP2]. If the sets $X_{1}, \ldots, X_{m}$ are separated in a "geometric", "algebraic" or "combinatorial" sense, then these sets are separated in all three senses.

Proof. We will prove that separated in an "algebraic" sense implies separated in a "geometric" sense, which implies separated in a "combinatorial" sense and which implies separated in an "algebraic" sense.

("algebraic" $\rightarrow$ "geometric") Let $x_{i} \in X_{i}$ and $x_{m}^{\prime} \in X_{m}$. We know that the orientation of $x_{1}, \ldots, x_{m-1}, x_{m}$ and $x_{1}, \ldots, x_{m-1}, x_{m}^{\prime}$ is the same, but then so are the orientations $x_{1}, \ldots, x_{m-1}, y_{m}$, where $y_{m} \in\left[x_{m}, x_{m}^{\prime}\right]$. This means that the sets $X_{1}, \ldots, X_{d}$, conv $X_{d+1}$ are also separated in an "algebraic" sense, and therefore the sets conv $X_{1}, \ldots$, conv $X_{m}$ too are separated in an "algebraic" sense. However, now a hyperplane intersecting at least $d+1$ of these convex hulls, contains $d+1$ points having orientation zero which is a contradiction.

("geometric" $\rightarrow$ "combinatorial") Choose $x_{i} \in X_{i}, 1 \leq i \leq m$, and an arbitrary hyperplane $H$, missing the points $x_{i}$. Let $I \subset[m]$ be the indices of the points $x_{i}$ on a given side of $H$. We need to show that

$$
\left(\operatorname{conv} \bigcup_{i \in I} X_{i}\right) \cap\left(\operatorname{conv} \bigcup_{i \in I^{c}} X_{i}\right)=\emptyset
$$

since then a hyperplane $H^{\prime}$ exists, which has all points of the set $X_{i}$ on one side for all $i$, and divide them in the same way as $H$ divides the points $x_{i}$. Suppose that

$$
\left(\operatorname{conv} \bigcup_{i \in I} X_{i}\right) \cap\left(\operatorname{conv} \bigcup_{i \in I^{c}} X_{i}\right) \neq \emptyset .
$$

Due to Radon's theorem there are $d+2$ points $y_{1}, \ldots, y_{d+2}$, we may suppose $y_{i} \in X_{i}$, such that

$$
\operatorname{conv} \bigcup_{i \in I} y_{i} \cap \operatorname{conv} \bigcup_{i \in I^{c}} y_{i} \neq \emptyset
$$

We call this a Radon-partition for the given points. If $d+2$ points are in general position, then the Radon-partition is well defined. Moving $y_{i}$ continuously toward $x_{i}$, the Radonpartition has to be unique and therefore unchanged. However, at the end we must have a different one to that at the beginning. This shows that our assumption that the intersection is not empty was wrong, so $H^{\prime}$ exists.

("combinatorial" $\rightarrow$ "geometric") Since $d+1$ points can be separated in any bipartition, so all $d+1$ sets can be separated. Therefore a hyperplane $H$ cannot meet $d+1$ sets conv $X_{1}, \ldots$, conv $X_{d+1}$ since there would be a Radon-partition according to this hyperplane for which no hyperplane would separate the sets into the parts of the Radon-partition. 


\section{References}

[BV] I. Bárány, P. Valtr, A positive fraction Erdôs-Szekeres theorem, Discrete Comput. Geom. 19 (1998), 335-342.

[EP] P. Erdős, G. Purdy, Extremal problems in combinatorial geometry, in: Handbook of Combinatorics (R. Graham, M. Grotschel, L. Lovász, eds.). Elsevier, New York, pp. 809-874, 1995.

[ES1] P. Erdős, Gy. Szekeres, A combinatorial problem in geometry, Compositio Math 2 (1935), 463-470.

[ES2] P. Erdős, Gy. Szekeres, On some extremum problems in elementary geometry, Ann. Univ. Sci. Budapest 3/4 (1960/61), 53-62.

[GP1] J. E. Goodman, R. Pollack, The complexity of point configurations, Discrete Appl. Math. 31 (1991), $167-180$.

[GP2] J. E. Goodman, R. Pollack, Allowable sequences and order types in discrete and computational geometry, in: New Trends in Discrete and Computational Geometry (J. Pach, ed.). Springer-Verlag, New York, pp. 103-134, 1993.

[K1] G. Kalai, Private communication, 1997.

[K2] Gy. Károlyi, Ramsey-remainder for convex sets and the Erdős-Szekeres theorem, Discrete Appl. Math. 1-2 (2001), 163-175.

[MM] M. Marcus, H. Minc, Convexity and matrices, in: A Survey of Matrix Theory and Matrix Inequalities, Allyn and Bacon, Boston, MA, pp. 121-138, 1964.

[N] M. J. Nielsen, Transverse matchings on a finite planar set, Manuscript, University of Idaho, Moscow, ID, 1995.

[PS] J. Pach, J. Solymosi, Canonical theorems for convex sets, Discrete Comput. Geom. 19 (1998), 427-435.

[PV] A. Pór, P. Valtr, Partitioned version of the Erdős-Szekeres theorem, Discrete Comput. Geom. 28 (2002), 625-637.

[S1] P. Schmitt, Problems in discrete and combinatorial geometry, in: Handbook of Convex Geometry (P. M. Gruber, J. M. Willis, eds.). Elsevier, New York, pp. 449-483, 1993.

[S2] J. Solymosi, Combinatorial problems in finite Ramsey theory, Master's thesis, Eötvös University, Budapest, 1988.

[TV] G. Tóth, P. Valtr, Note on the Erdős-Szekeres theorem, Discrete Comput. Geom. 19 (1998), 457-459.

Received November 27, 2000, and in revised form April 5, 2002, September 26, 2002, and November 15, 2002.

Online publication July 15, 2003. 